\title{
Search for Deconfinement in NA49 at the CERN SPS
}

\author{
Peter Seyboth, Max-Planck-Institut für Physik, 80805 Munich, Germany
}

for the NA49 collaboration:

S.V. Afanasiev ${ }^{9}$, T. Anticic ${ }^{19}$, D. Barna ${ }^{5}$, J. Bartke ${ }^{7}$, R.A. Barton ${ }^{3}$, L. Betev ${ }^{10}$, H. Białkowska $^{17}$, A. Billmeier ${ }^{10}$, C. Blume ${ }^{8}$, C.O. Blyth ${ }^{3}$, B. Boimska ${ }^{17}$, M. Botje ${ }^{1}$, J. Bracinik ${ }^{4}$, R. Bramm ${ }^{10}$, R. Brun ${ }^{11}$, P. Bunčić ${ }^{10,11}$, V. Cerny ${ }^{4}$, O. Chvala ${ }^{15}$, J.G. Cramer ${ }^{16}$, P. Csató ${ }^{5}$, P. Dinkelaker ${ }^{10}$, V. Eckardt ${ }^{14}$, P. Filip ${ }^{14}$, H.G. Fischer ${ }^{11}$, Z. Fodor ${ }^{5}$, P. Foka ${ }^{8}$, P. Freund ${ }^{14}$, V. Friese ${ }^{13}$, J. Gál ${ }^{5}$, M. Gaździcki ${ }^{10}$, G. Georgopoulos ${ }^{2}$, E. Gładysz ${ }^{7}$, S. Hegyi ${ }^{5}$, C. Höhne ${ }^{13}$, P.G. Jones ${ }^{3}$, K. Kadija ${ }^{11,19}$, A. Karev ${ }^{14}$, V.I. Kolesnikov ${ }^{9}$, T. Kollegger ${ }^{10}$, M. Kowalski ${ }^{7}$, I. Kraus ${ }^{8}$, M. Kreps ${ }^{4}$, M. van Leeuwen ${ }^{1}$, P. Lévai ${ }^{5}$, A.I. Malakhov ${ }^{9}$, C. Markert ${ }^{8}$, B.W. Mayes ${ }^{12}$, G.L. Melkumov ${ }^{9}$, A. Mischke ${ }^{8}$,J. Molnár ${ }^{5}$, J.M. Nelson ${ }^{3}$, G. Pálla ${ }^{5}$, A.D. Panagiotou ${ }^{2}$, K. Perl ${ }^{18}$, A. Petridis ${ }^{2}$, M. Pikna ${ }^{4}$, L. Pinsky ${ }^{12}$, F. Pühlhofer ${ }^{13}$, J.G. Reid ${ }^{16}$, R. Renfordt ${ }^{10}$, W. Retyk ${ }^{18}$, C. Roland ${ }^{6}$, G. Roland ${ }^{6}$, A. Rybicki ${ }^{7}$, A. Sandoval ${ }^{8}$, H. Sann ${ }^{8}$, N. Schmitz ${ }^{14}$, P. Seyboth ${ }^{14}$, F. Siklér ${ }^{5}$, B. Sitar ${ }^{4}$, E. Skrzypczak ${ }^{18}$, G.T.A. Squier ${ }^{3}$, R. Stock ${ }^{10}$, H. Ströbele ${ }^{10}$, T. Susa ${ }^{19}$, I. Szentpétery ${ }^{5}$, J. Sziklai ${ }^{5}$, T.A. Trainor ${ }^{16}$, D. Varga ${ }^{5}$, M. Vassiliou ${ }^{2}$, G.I. Veres ${ }^{5}$, G. Vesztergombi ${ }^{5}$, D. Vranić ${ }^{8}$, S. Wenig ${ }^{11}$, A. Wetzler ${ }^{10}$, I.K. Yoo ${ }^{13}$, J. Zaranek $^{10}$, J. Zimányi ${ }^{5}$

${ }^{1}$ NIKHEF, Amsterdam, Netherlands.

${ }^{2}$ Department of Physics, University of Athens, Athens, Greece.

${ }^{3}$ Birmingham University, Birmingham, England.

${ }^{4}$ Comenius University, Bratislava, Slovakia.

${ }^{5}$ KFKI Research Institute for Particle and Nuclear Physics, Budapest, Hungary.

${ }^{6} \mathrm{MIT}$, Cambridge, USA.

${ }^{7}$ Institute of Nuclear Physics, Cracow, Poland.

${ }^{8}$ Gesellschaft für Schwerionenforschung (GSI), Darmstadt, Germany.

${ }^{9}$ Joint Institute for Nuclear Research, Dubna, Russia.

${ }^{10}$ Fachbereich Physik der Universität, Frankfurt, Germany.

${ }^{11}$ CERN, Geneva, Switzerland.

${ }^{12}$ University of Houston, Houston, TX, USA.

${ }^{13}$ Fachbereich Physik der Universität, Marburg, Germany.

${ }^{14}$ Max-Planck-Institut für Physik, Munich, Germany.

${ }^{15}$ Institute of Particle and Nuclear Physics, Charles University, Prague, Czech Republic.

${ }^{16}$ Nuclear Physics Laboratory, University of Washington, Seattle, WA, USA.

${ }^{17}$ Institute for Nuclear Studies, Warsaw, Poland.

${ }^{18}$ Institute for Experimental Physics, University of Warsaw, Warsaw, Poland.

${ }^{19}$ Rudjer Boskovic Institute, Zagreb, Croatia. 
Abstract: Experiment NA49 at the Cern SPS uses a large acceptance detector for a systematic study of particle yields and correlations in nucleus-nucleus, nucleon-nucleus and nucleon-nucleon collisions. Preliminary results for $\mathrm{Pb}+\mathrm{Pb}$ collisions at 40, 80 and $158 \mathrm{~A} \cdot \mathrm{GeV}$ beam energy are shown and compared to measurements at lower and higher energies.

\section{Introduction}

The primary purpose of the heavy ion programme at the CERN SPS is the search for evidence of a transient deconfined state of strongly interacting matter during the early stage of nucleus-nucleus collisions [1]. The transition from a dilute state of individual hadrons to a phase of quasi-free quarks and gluons, the quark gluon plasma (QGP), was first suggested using qualitative arguments [2] and later confirmed by quantum chromodynamics (QCD) on the lattice provided the energy density reaches sufficiently high values in an extended volume.

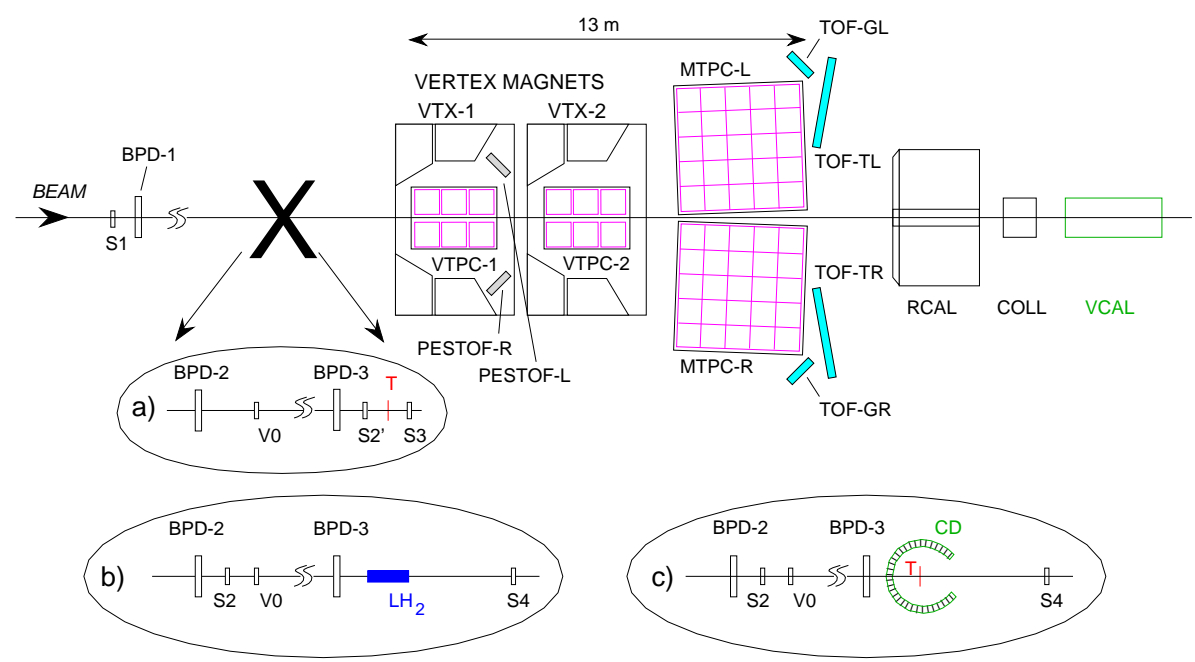

Figure 1: Schematic layout of the NA49 experiment at the Cern SPS showing beam detectors, superconducting dipole magnets, time projection chambers (VTPC,MTPC), time-of-flight arrays (TOF) and calorimeters (RCAL,VCAL). A thin solid target $\mathrm{T}$ is used for $\mathrm{A}+\mathrm{A}$ collisions (a), which is surrounded by a detector of slow protons (CD) for $\mathrm{p}+\mathrm{A}$ collsions (c). A liquid $\mathrm{H}_{2}$ target is employed for $\mathrm{p}+\mathrm{p}$ collisions (b)

The results from the heavy-ion program at CERN in fact indicated that deconfinement may set in within the SPS energy range [3]. Within most model scenarios the data imply that the initial energy density exceeds the critical value. Originally proposed signatures [4] of the QGP were observed in $\mathrm{Pb}+\mathrm{Pb}$ collisions at the top SPS energy, i.e. $J / \Psi$ suppression, strangeness enhancement, and possibly thermal photons and dileptons. The significance of these signals as QGP signatures has come under renewed scrutiny. Moreover, there is no observational evidence for a sharp phase transition from QGP to hadrons such as 
phase coexistence [5], critical fluctuations [5, 6] or more speculative effects like DCC [7] or parity violation [8].

The NA49 experiment is performing an energy scan from $20-158 \mathrm{~A} \cdot \mathrm{GeV}$ in an effort to strengthen the evidence for the onset of deconfinement by searching for anomalies in the energy dependence of experimental observables. Data at 40, 80 and $158 \mathrm{~A} \cdot \mathrm{GeV}$ have so far been recorded and analysed. A brief description of the apparatus (section 2) is followed by preliminary results for $\pi, \mathrm{K}, \Lambda$ and $\bar{\Lambda}$ yields, $\pi \pi$ correlations, event-to-event charge fluctuations, anisotropic flow (sections 3 - 6), and conclusions (section 7).

\section{The NA49 detector}

The NA49 experiment [9] was designed for the investigation of hadron production in the most violent $\mathrm{Pb}+\mathrm{Pb}$ interactions at the Cern SPS. The main features (Fig.I) are large acceptance precision tracking and particle identification using time projection chambers (TPCs). The first two are located inside superconducting dipole magnets which provide the particle trajectory bending necessary for momentum determination. Charged particles in the forward hemisphere of the reaction are identified from the measurement of their energy loss $\mathrm{dE} / \mathrm{dx}$ in the TPC gas (accuracy $3-4 \%$ ). At central rapidity the identification is further improved by measurement of the time-of-flight (resolution $60 \mathrm{ps)} \mathrm{to} \mathrm{arrays} \mathrm{of}$ scintillation counter tiles (TOF-T) and strips (TOF-G). Strange particles are detected via decay topology and invariant mass measurement. The forward calorimeter VCAL measures the energy of the projectile spectators from which one can deduce the impact parameter in $\mathrm{A}+\mathrm{A}$ collisions.
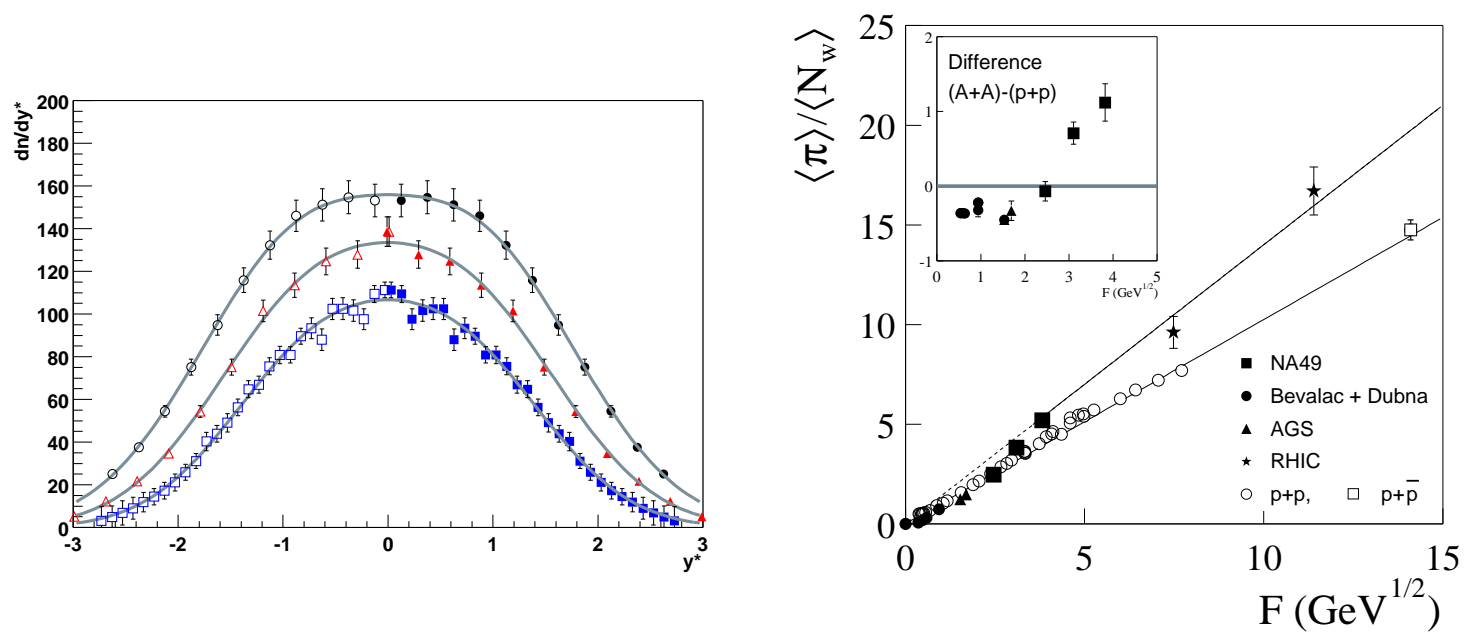

Figure 2: Left: rapidity distribution of $\pi^{-}$in central $\mathrm{Pb}+\mathrm{Pb}$ collisons at 40 (squares), 80 (triangles) and 158 (dots) A.GeV. Open symbols show values reflected at $\mathrm{y}^{\star}=0$ (NA49 preliminary). Right: total pion multiplicity $\langle\pi\rangle$ produced per wounded (participant) nucleon versus the Fermi energy variable $\mathrm{F} \approx s_{N N}^{0.25}$ for $\mathrm{p}+\mathrm{p}$ reactions (open symbols) and central nucleus-nucleus collisions (full symbols). 


\section{$3 \quad$ Yields of $\pi, \mathbf{K}, \Lambda$ and $\bar{\Lambda}$}

Raw $\mathrm{K}^{+}$and $\mathrm{K}^{-}$yields were extracted from fits of the distributions of $\mathrm{dE} / \mathrm{dx}$ and TOF (where available) in narrow bins of rapidity y and transverse momentum $\mathrm{p}_{T}$. The resulting spectra were then corrected for geometrical acceptance, losses due to in-flight decays and reconstruction efficiency. The latter was determined from embedding simulated tracks into real events and amounted to $\approx 95 \%$. Spectra of $\pi^{-}$mesons were derived from the acceptance corrected negatively charged particle yields in $\mathrm{p}_{T}$ and $\mathrm{y}$ (assuming the $\pi$ mass) by subtracting the estimated contribution of $\mathrm{K}^{-}, \overline{\mathrm{p}}$ and the contamination from secondary hadron decays. The ratio $\pi^{+} / \pi^{-}$was determined in the region where both $\mathrm{dE} / \mathrm{dx}$ and TOF are available $(0.91,0.94$ and 0.97 at 40,80 and $158 \mathrm{~A} \cdot \mathrm{GeV})$ and was assumed to be y independent.
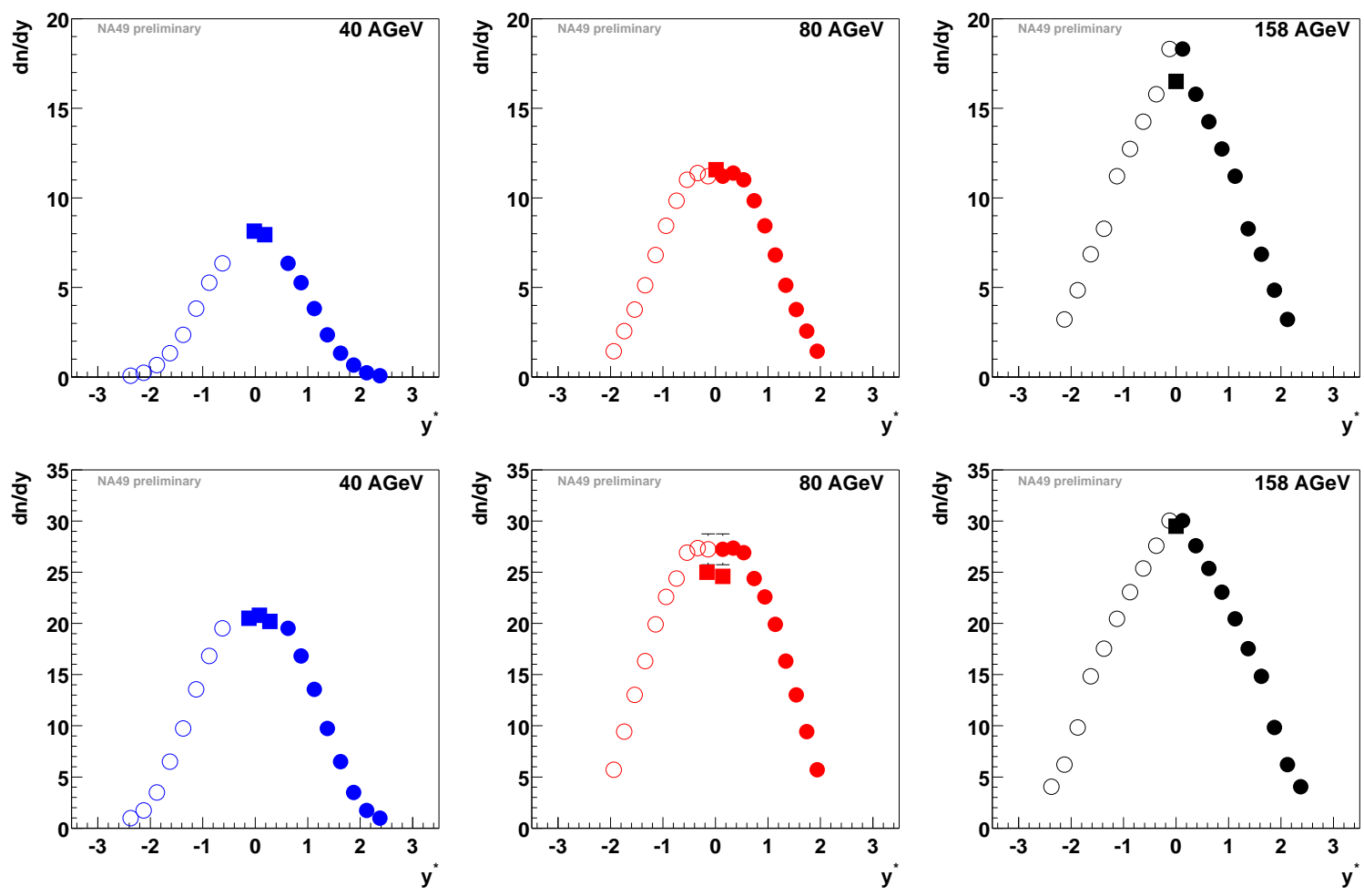

Figure 3: Rapidity distribution of $\mathrm{K}^{-}$(top row) and $\mathrm{K}^{+}$(bottom row) from $\mathrm{dE} / \mathrm{dx}$ (circles) and combined $\mathrm{dE} / \mathrm{dx}$ and TOF (squares) analysis. Open symbols show values reflected at $\mathrm{y}^{\star}=0$. (NA49 preliminary)

The resulting rapidity distributions of $\pi^{-}$are plotted in Fig.2, left. The integrated yields are $312 \pm 15,445 \pm 22$ and $610 \pm 30$ at 40,80 and $158 \mathrm{~A} \cdot \mathrm{GeV}$ respectively. Pions are the dominant produced particle species and thus their number provides a measure of the entropy in a statistical model description of the reaction. The yield of pions (estimated here as $\left.\langle\pi\rangle=1.5 \cdot\left(\left\langle\pi^{-}\right\rangle+\left\langle\pi^{+}\right\rangle\right)\right)$divided by the number of wounded nucleons (participants) $\mathrm{N}_{W}$ is shown versus the Fermi energy variable $\mathrm{F} \equiv\left(\sqrt{s}-2 m_{N}\right)^{3 / 4} /{\sqrt{s_{N N}}}^{1 / 4} \approx s_{N N}^{1 / 4}$ in 
Fig.2, right. While $\mathrm{p}+\mathrm{p}$ data show a linear rise throughout there is a change for $\mathrm{A}+\mathrm{A}$ collisions (illustrated more clearly in the inset) in the SPS energy range. Below one finds a regime of slight suppression, above a region of enhancement with a steeper linear rise than for $\mathrm{p}+\mathrm{p}$ reactions. This steepening has been interpreted as indicating the activation of a large number of partonic degrees of freedom at the onset of deconfinement [10].
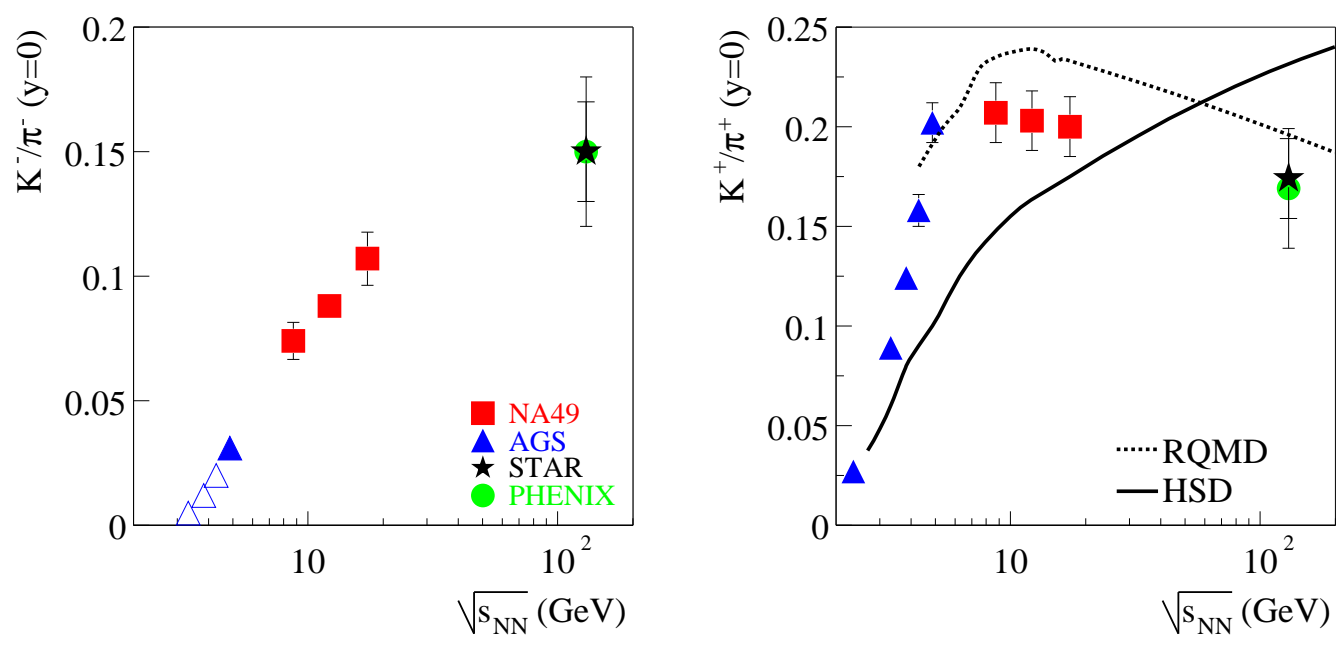

Figure 4: Midrapidity ratio of $\mathrm{K}^{-} / \pi^{-}$(left) and $\mathrm{K}^{+} / \pi^{+}$(right) as function of energy from NA49 (squares, preliminary) compared to measurements at lower and higher energies. Predictions of the RQMD [11] (dotted) and HSD [12] (full curve) models are shown.

Kaons contain about $75 \%$ of the $s, \bar{s}$ quarks in the produced hadrons at SPS energies and thus their number indicates the total strangeness content of the final state. The rapidity distributions are displayed in Fig. 3 and integrate with small extrapolation to total yields of $18 \pm 1,29 \pm 2,50 \pm 5$ for $\mathrm{K}^{-}$and $56 \pm 3,79 \pm 5,95 \pm 9$ for $\mathrm{K}^{+}$at $40,80,160$ A. GeV respectively. Yields of most particles, of course, increase with energy. Changes in the composition of the produced system are better characterised by particle ratios. Measurements of $\mathrm{K}^{-} / \pi^{-}$and $\mathrm{K}^{+} / \pi^{+}$at midrapidity from NA49 are plotted versus energy in Fig.t and compared to results at lower and higher energies.

A continuous increase is seen for $\mathrm{K}^{-} / \pi^{-}$. For $\mathrm{K}^{+} / \pi^{+}$one finds a steeper rise followed by a maximum at the lower end of the SPS energy region and a gradual decrease. Within a reaction scenario based on nucleon-nucleon collisions these features might be attributed to thresholds and the decrease of the baryon density with increasing energy. The ratio $\mathrm{K}^{-} / \pi^{-}$exhibits the threshold of the $\mathrm{KK}$ production mechanism. The lower mass threshold of associate $\mathrm{KY}$ production leads to a steeper rise in $\mathrm{K}^{+} / \pi^{+}$and the rapidly falling baryon density may result in a compensation of the declining contribution from the KY by the growing contribution from the KK production mechanism. However, the continuous increase seen for nucleon-nucleon collisions (e.g. Fig.7, right) does not really support such an interpretation.

The most abundantly produced hyperons are $\Lambda$ and $\bar{\Lambda}$ for which Fig. 5 shows the rapidity distributions. These show a broad shape for $\Lambda$ reflecting the associate production 

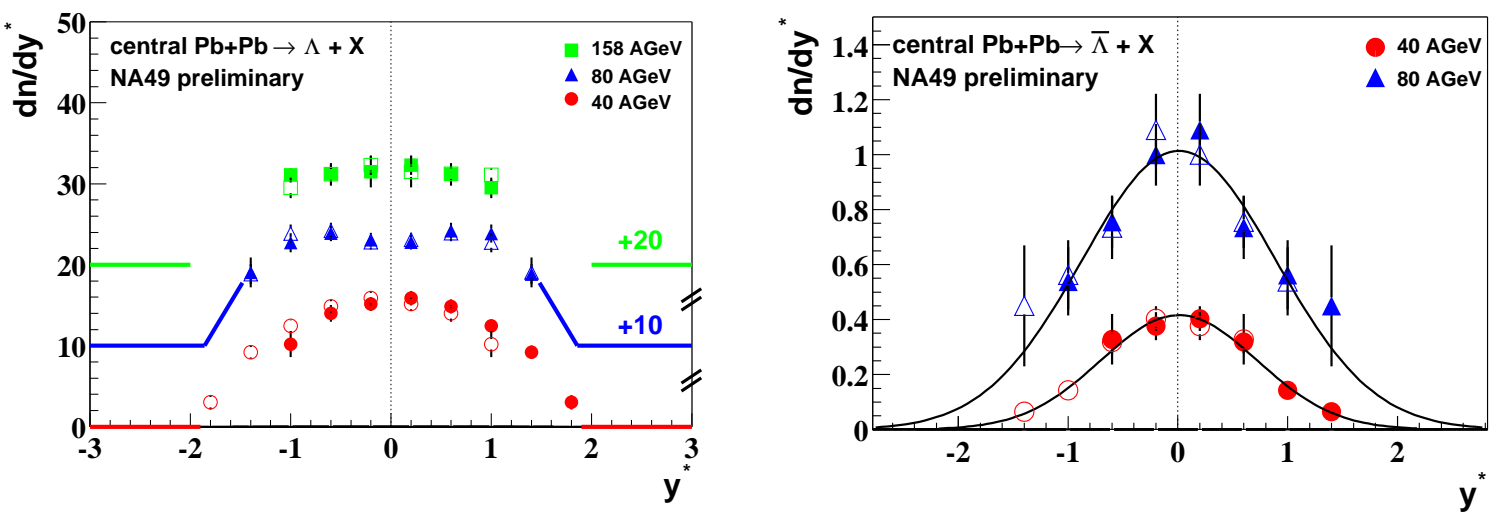

Figure 5: Rapidity distribution of $\Lambda$ (left) and $\bar{\Lambda}$ (right). Open symbols show values reflected at $\mathrm{y}^{\star}=0 . \Lambda$ yields at 80 and $158 \mathrm{~A} \cdot \mathrm{GeV}$ are displaced vertically by 10 respectively 20 units for clarity. (NA49 preliminary)

mechanism and the partially stopped participant nucleon distribution. In contrast the distribution is of narrower Gaussian type for $\bar{\Lambda}$ which are most likely produced as members of hyperon-antihyperon $(\mathrm{Y} \bar{Y})$ pairs. The ratios $\langle\Lambda\rangle /\langle\pi\rangle$ and $\langle\bar{\Lambda}\rangle /\langle\pi\rangle$ of $4 \pi$ integrated yields are displayed as a function of energy in Fig.6. One observes a steep threshold rise for the $\langle\Lambda\rangle /\langle\pi\rangle$ ratio followed by a decline which can be mainly attributed to the rapidly decreasing net baryon density. The ratio $\langle\bar{\Lambda}\rangle /\langle\pi\rangle$ exhibits a continuous rise due to the high mass $Y \bar{Y}$ threshold.
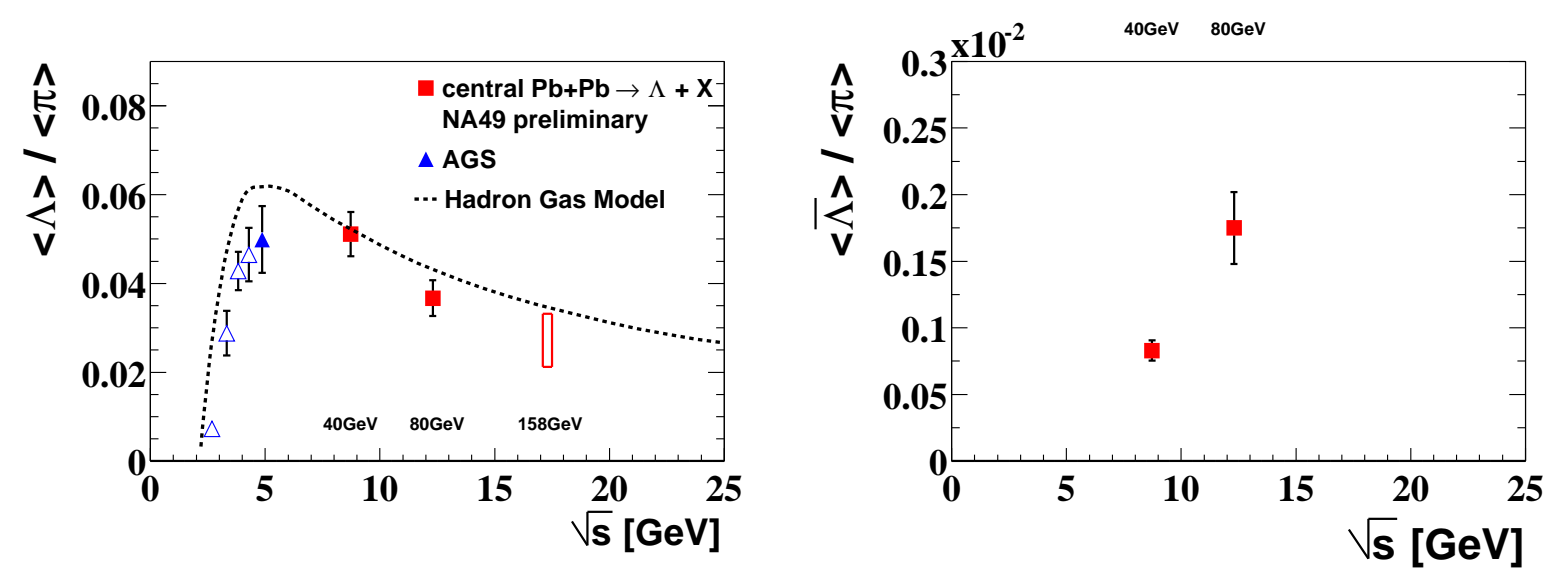

Figure 6: $4 \pi$ yield ratios $\langle\Lambda\rangle /\langle\pi\rangle$ (left) and $\langle\bar{\Lambda}\rangle /\langle\pi\rangle$ (right) versus energy from NA49 (squares, preliminary) and lower energy AGS experiments. The dotted line shows a prediction from the extended hadron gas model [13]. 
Microscopic dynamical and statistical models have been used extensively to describe particle yields in a wide variety of reactions. The first class is often based on string excitation, fusion and hadronisation (e.g. HSD [12], RQMD [11], UrQMD [14]) followed by reinteractions of the formed hadrons (RQMD, UrQMD). As seen from Fig. 1 HSD does not reproduce the energy dependence of the midrapidity $\mathrm{K}^{+} / \pi^{+}$ratio. On the other hand, RQMD correctly predicts the trend, but somewhat overpredicts the ratio in the SPS energy range.

A comparison with the $4 \pi$ ratio $\left\langle\mathrm{K}^{+}\right\rangle /\left\langle\pi^{+}\right\rangle$is presented in Fig.7 left. Both UrQMD and RQMD get the steep threshold rise. UrQMD values are much too low in the plateau due to an overprediction of pions. RQMD does not follow the drop in the SPS range which is indicated by the measurements.
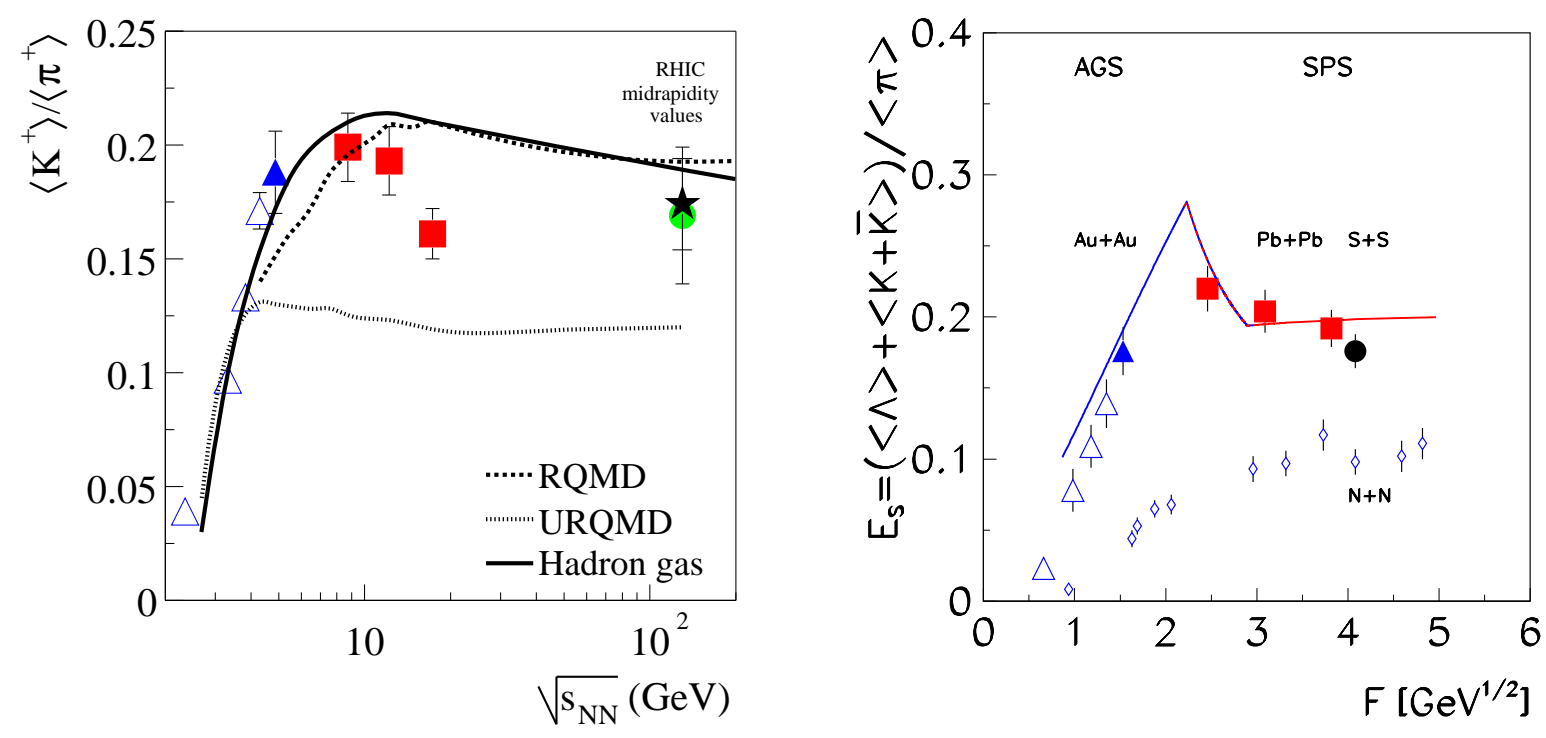

Figure 7: Left: $4 \pi$ ratio $\left\langle\mathrm{K}^{+}\right\rangle /\left\langle\pi^{+}\right\rangle$versus energy compared to predictions of the RQMD [11] (dashed), UrQMD [14] (dotted) and extended statistical [13] (solid curve) models. Right: the strangeness content measure $\mathrm{E}_{s}$ versus the Fermi energy variable $\mathrm{F} \approx s_{N N}^{0.25}$ compared to the prediction of the statistical model of the early stage [10] (curves).

Since antihyperon production rates are small and isospin symmetry approximately holds $\left(\left\langle\mathrm{K}^{+}\right\rangle \approx\left\langle\mathrm{K}^{0}\right\rangle\right)$ nearly half of the $\bar{s}$ quarks in the produced hadrons are contained in $\mathrm{K}^{+}$mesons. Moreover, strangeness conservation requires $\langle s\rangle=\langle\bar{s}\rangle$. Thus $\left\langle\mathrm{K}^{+}\right\rangle$measures to a good approximation one quarter of all $s$ and $\bar{s}$ quarks in the final state hadrons. The energy dependence of the $\left\langle\mathrm{K}^{+}\right\rangle /\left\langle\pi^{+}\right\rangle$ratio (see Fig.7 left) therefore indicates a maximum in the fraction of strangeness carrying particles in the lower SPS energy range.

Statistical models have been surprisingly successful in describing ratios of particle yields in many types of reactions over a wide energy range. Since the widths of rapidity distributions depend on particle mass in the SPS energy range and below, the model should preferentially be compared to $4 \pi$ yields. Fits of this model to NA49 data [15] have indicated that while there is relative hadro-chemical equilibrium in the strange 
particle sector there seems to be an overall undersaturation with respect to non-strange particle yields. The statistical model as such makes no prediction concerning the energy dependence of particle production. However, it has recently been supplemented [13] by a parameterisation of the energy dependence of its two main parameters, baryochemical potential $\mu_{B}$ and temperature T. Predictions are shown in Fig.7 left for $\left\langle\mathrm{K}^{+}\right\rangle /\left\langle\pi^{+}\right\rangle$and Fig.6 right for $\langle\Lambda\rangle /\langle\pi\rangle$. The trend of the data is well reproduced by this extended statistical model calculation. In detail the decrease in the SPS energy range of both $\left\langle\mathrm{K}^{+}\right\rangle /\left\langle\pi^{+}\right\rangle$ and $\langle\Lambda\rangle /\langle\pi\rangle$ and even more so of $\langle\Xi\rangle /\langle\pi\rangle$ (not shown) is not well described.

Predictions have also been published for a statistical model which explicitly assumes a deconfined phase in the early stage above a certain threshold energy [10]. In this model the strangeness to entropy ratio is assumed to be established initially and to persist through the hadronisation stage. A measure of this quantity is the ratio $\mathrm{E}_{s}=(\langle\Lambda\rangle+\langle K+\bar{K}\rangle) /\langle\pi\rangle$ evaluated from the final hadron multiplicities which is plotted in Fig. 7 right. After a rise corresponding to the purely hadronic reaction in the model, one observes a saturation at the a level consistent with the strangeness to entropy ratio expected for an initially deconfined system.
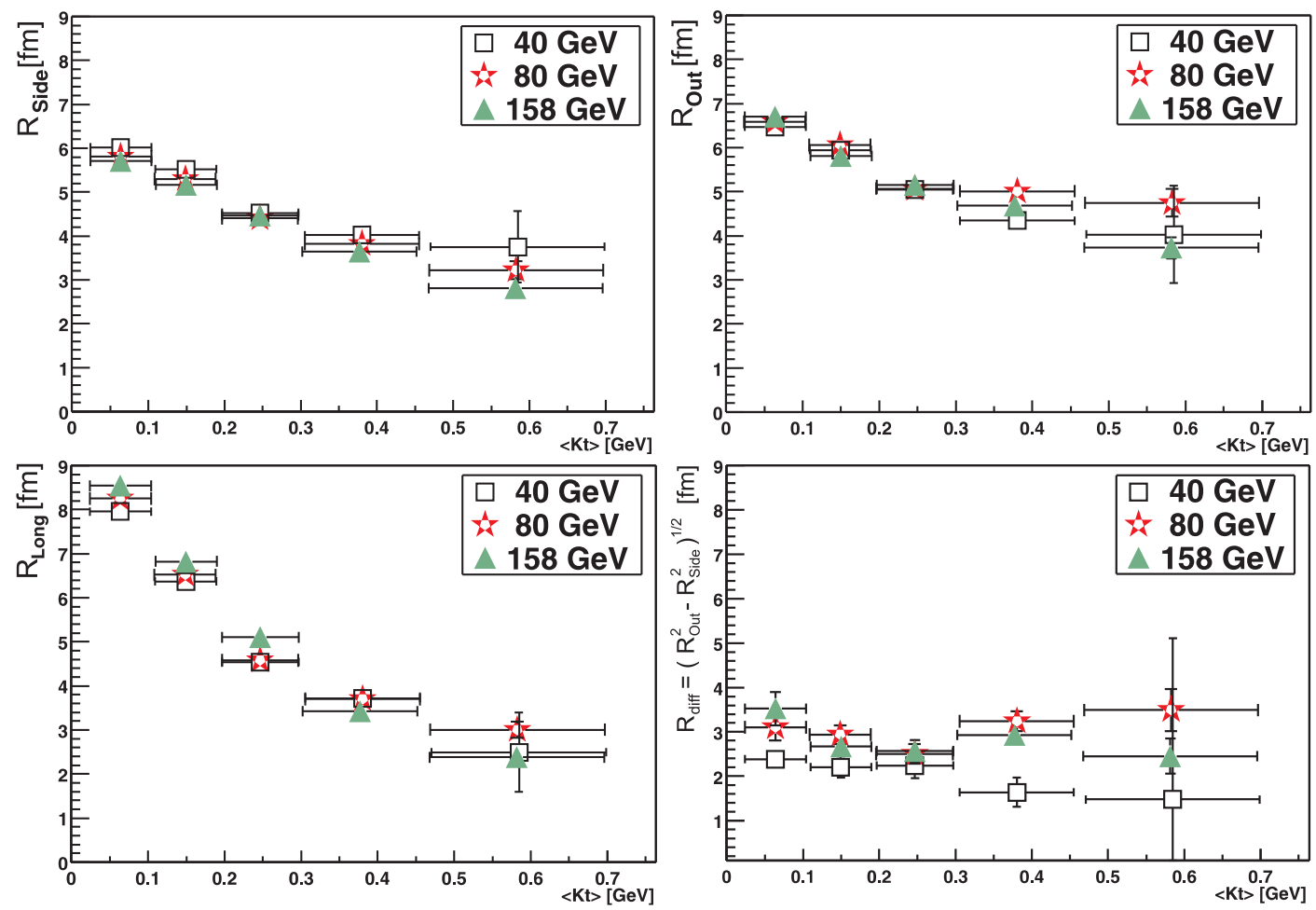

Figure 8: Gaussian radius parameters $\mathrm{R}_{\text {side }}, \mathrm{R}_{\text {out }}, \mathrm{R}_{\text {long }}$ fitted to the $\pi^{-} \pi^{-}$correlation function evaluated in the longitudinally comoving frame plotted versus the average transverse momentum $\mathrm{K}_{T}$ of the pair. Rapidity range $\mathrm{y}^{\star} \leq \mathrm{y} \leq \mathrm{y}^{\star}+0.5$ (NA49 preliminary). 


\section{$4 \pi \pi$ correlations}

Correlations of pions with near equal momenta $\mathbf{p}_{1}, \mathbf{p}_{2}$ provide information on the size and internal dynamics of the fireball source at freezout [16]. The analysis was performed in the longitudinally comoving reference frame, decomposing the momentum difference $\mathrm{Q}=\mathbf{p}_{1}-\mathbf{p}_{2}$ into long, side, out components. For $\pi^{+} \pi^{-}$pairs the correlation peak at small $\mathrm{Q}$ is predominantly due to the Coulomb attraction and can be well reproduced by a Coulomb wave calculation [17] using the measured effective source size. Correlations of $\pi^{-} \pi^{-}$are described by a product of parameterised Coulomb repulsion [17] and the quantum statistics enhancement, fitted with a Gaussian parameterisation. The resulting radius parameters $\mathrm{R}_{\text {side }}, \mathrm{R}_{\text {out }}, \mathrm{R}_{\text {long }}$ are plotted in Fig. 8 versus the average transverse momentum $\mathrm{K}_{T}$ of the pair. No striking energy dependence is observed. Within expanding source models the decrease with $\mathrm{K}_{T}$ is a manifestation of the strong longitudinal $\left(\mathrm{R}_{\text {long }}\right)$ and radial $\left(\mathrm{R}_{\text {side }}, \mathrm{R}_{\text {out }}\right)$ flow at SPS energies. Moreover, there is little change in the lifetime $\tau_{0} \approx \mathrm{R}_{\text {long }} \sqrt{T / M_{T}}$ or the emission duration $\Delta \tau^{2} \approx\left(\mathrm{R}_{\text {out }}^{2}-\mathrm{R}_{\text {side }}^{2}\right) / \beta_{T}^{2}$ of the source. The small value of $\Delta \tau$ does not indicate a soft point of the matter equation of state of the kind discussed in ref. [5] near the onset of deconfinement.
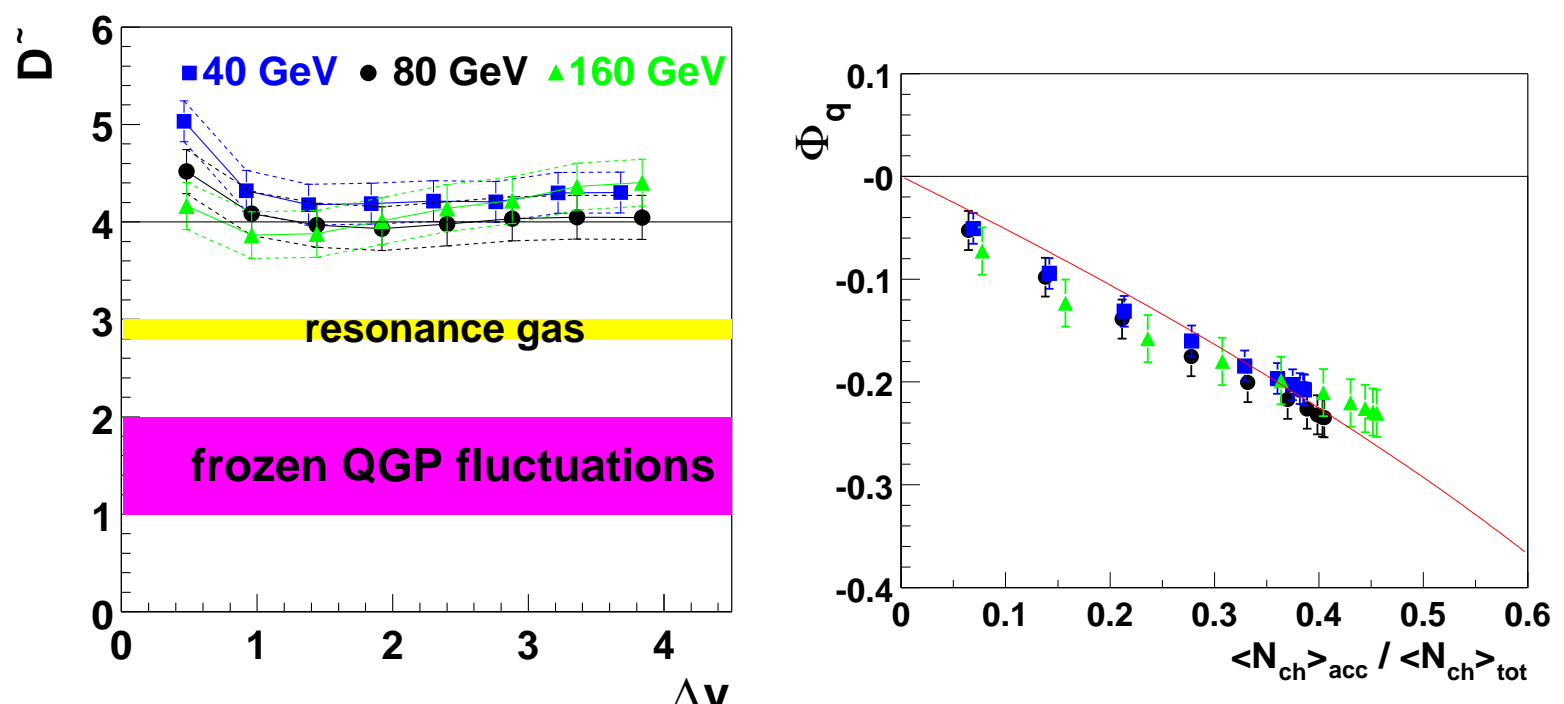

Figure 9: Measures of event-to-event charge fluctuations in central $\mathrm{Pb}+\mathrm{Pb}$ collisions. Left: $\tilde{D}$ versus the size of the rapidity window $\Delta y$. Right: $\Phi_{q}$ versus the ratio $\left\langle N_{c h}\right\rangle /\left\langle N_{c h}\right\rangle_{t o t}$ of the multiplicity in the acceptance window and the total multiplicity in the events; the curve shows the prediction for independent particle emission plus global charge conservation (NA49 preliminary). 


\section{Event-to-event charge fluctuations}

Recently it was proposed that event-to-event fluctuations of the charge ratio $\mathrm{R}=\mathrm{N}_{+} / \mathrm{N}_{-}$ or the net charge $\mathrm{Q}=\mathrm{N}_{+}-\mathrm{N}_{-}$may be sensitive to deconfinement in the early stage of nucleus-nucleus collisions [18, 19]. The smaller charge quanta in a partonic phase are expected to result in a reduction of such fluctuations.

The fluctuations of the charge ratio were investigated via the measure $\tilde{D}$ [20] which is corrected for the residual net charge in the considered rapidity interval $\Delta y$ as well as for global charge conservation. The preliminary NA49 results are shown in Fig.9 left and are found to be close to the expectation for independent particle emission plus global charge conservation $(\tilde{D} \approx 4)$ and do not change significantly with energy. No evidence is seen for the reduction predicted for a resonance gas nor for the large decrease expected for a QGP phase. It is, of course, not clear whether reduced fluctuations in the QGP will persist through the hadronisation, rescattering and resonance decay stages.

The quantity $\Phi_{q}$ was proposed in ref. [21] for studying fluctuations of the net charge. It is independent of the number of superimposed particle sources, has value zero for independent particle emission and -1 for local charge conservation. Preliminary NA49 measurements are plotted in Fig.9 right versus the ratio $\left\langle N_{c h}\right\rangle /\left\langle N_{c h}\right\rangle_{t o t}$ of the multiplicity in the acceptance window and the total multiplicity in the events. Again no significant energy dependence is observed and the results are close to the prediction for independent particle emission plus global charge conservation $\Phi_{q}^{c c}=\sqrt{1-\left\langle N_{c h}\right\rangle /\left\langle N_{c h}\right\rangle_{t o t}}-1$ (ref. [22], line in Fig.9).
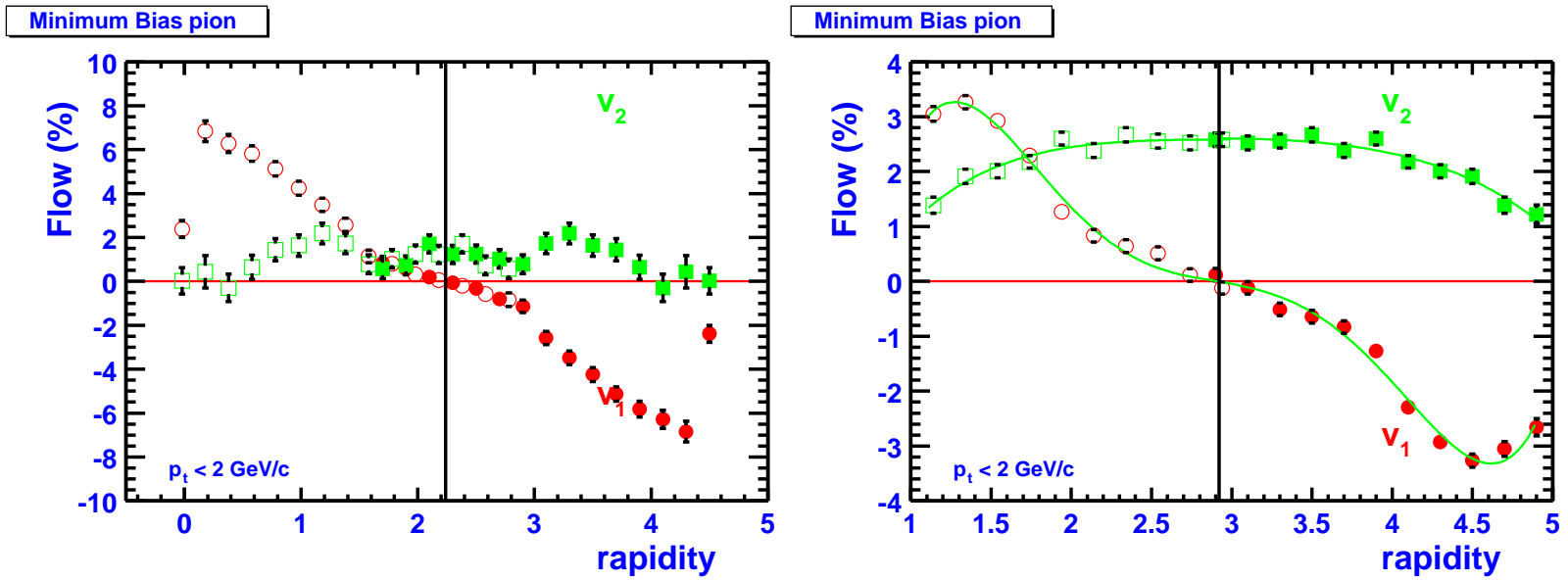

Figure 10: Fourier coefficients $v_{1}$ (directed flow) and $v_{2}$ (elliptic flow) of the azimuthal distribution of pions in min.bias $\mathrm{Pb}+\mathrm{Pb}$ collisions versus the rapidity at 40 (left) and 158 (right) A.GeV beam energy (NA49 preliminary). 


\section{Anisotropic flow}

Anisotropic flow in non-central collisions is sensitive to pressure in the early stage of the reaction, which can transform the initial space anisotropy of the reaction zone into an azimuthal anisotropy of the momentum distribution of the observed particles. The onset of deconfinement might result in a minimum of this effect [5]. Anisotropic flow is quantified by the Fourier coefficients $v_{n}$ of the distribution of particle azimuthal angles $\Phi$ with respect to the reaction plane $\Psi$ [23]:

$$
v_{n}=\left\langle\cos \left(n\left(\Phi_{i}-\Psi_{n}\right)\right)\right\rangle=\sqrt{2}\left\langle\sin \left(n \cdot \Phi_{i}\right) \cdot \sin \left(n \cdot \Psi_{n}\right)\right\rangle
$$

Corrections for reaction plane resolution, nonuniform azimuthal acceptance and momentum conservation were applied. The results for $v_{1}$ (directed flow) and $v_{2}$ (elliptic flow) for pions are plotted versus rapidity in Fig.10. The values for $v_{1}$ decrease from 40 (left) to 158 (right) A.GeV by a factor of 2 , whereas $v_{2}$ shows a slight increase.

\section{Conclusion}

The study of central $\mathrm{Pb}+\mathrm{Pb}$ collisions in $\mathrm{NA} 49$ at 40,80 and $158 \mathrm{~A} \cdot \mathrm{GeV}$ led to the following conclusions:

- the produced number of pions per participant in $\mathrm{Pb}+\mathrm{Pb}$ collisions changes from suppression with repsect to $\mathrm{p}+\mathrm{p}$ reactions to enhancement in the SPS energy range

- the fraction of produced particles containing $s$ or $\bar{s}$ quarks passes through a maximum at low SPS energies

- strangeness production starts to be undersaturated with respect to statistical equilibrium at SPS energies at a level consistent with the deconfinement hypothesis

- no unusual features are found in the evolution of other characteristics of the produced hadron system

NA49 will close the data gap between existing measurements at the AGS and the SPS with runs at 20 and $30 \mathrm{~A} \cdot \mathrm{GeV}$ in 2002.

\section{Acknowledgements}

This work was supported by the Director, Office of Energy Research, Division of Nuclear Physics of the Office of High Energy and Nuclear Physics of the US Department of Energy (DE-ACO3-76SFOOO98 and DE-FG02-91ER40609), the US National Science Foundation, the Bundesministerium fur Bildung und Forschung, Germany, the Alexander von Humboldt Foundation, the UK Engineering and Physical Sciences Research Council, the Polish State Committee for Scientific Research (5 P03B 13820 and 2 P03B 02418), the Hungarian Scientific Research Foundation (T14920 and T23790), the EC Marie Curie Foundation, and the Polish-German Foundation. 


\section{References}

[1] for review see Proceedings of the 14th International Conference on UltraRelativistic Nucleus-Nucleus Collisions, Quark Matter 99, eds.: L. Riccati, M. Masera and E. Vercellin, Nucl. Phys. A661 (1999) 1c.

[2] J. C. Collins and M. J. Perry, Phys. Rev. Lett. 34 (1975) 151.

[3] see: http://cern.web.cern.ch/CERN/Announcements/2000/NewStateMatter/

[4] review of QGP signatures: J.Harris and B.Müller, Annu. Rev. Nucl. Part. Sci. 46 (1996) 71.

[5] C.Hung and E.Shuryak, Phys. Rev. Lett. 75 (1995) 4003.

[6] M.Stephanov, K.Rajagopal and E.Shuryak, Phys. Rev. D60 (1999) 114028.

[7] K.Rajagopal and F.Wilczek, Nucl. Phys. 399 (1993) 395; B404 (1993) 557.

[8] D.Kharzeev, R.Pisarski and M.Tytgat, Phys. Rev. Lett. 81 (1998) 512.

[9] S.Afanasev et al., Nucl. Inst. Meth. A430 (1999) 210.

[10] M. Gaździcki and M. I. Gorenstein, Acta Phys. Polon. B30 (1999) 2705, and references therein.

[11] F. Wang, H. Liu, H. Sorge, N. Xu and J. Yang, Phys. Rev. C61 (2000) 064904.

[12] W. Cassing, E.L. Bratkovskaya and S. Juchem, Nucl. Phys. A674 (2000) 249.

[13] J. Cleymans and K. Redlich, Phys. Rev. C60 (1999) 054908,

P. Braun-Munzinger et al., Nucl. Phys. A697 (2002) 902.

[14] S. A. Bass et al., Prog. Part. Nucl. Phys. 41 (1998) 225; H. Weber et al. (UrQMD Collab.), to be published.

[15] F.Becattini, M.Gaździcki and J.Sollfrank, Eur. Phys. J. C5 (1998) 143.

[16] U.Wiedemann and U.Heinz, Phys. Rept. 319 (1999) 145.

[17] Y.Sinyukov et al., Phys. Lett. B432 (1998) 248.

[18] S.Jeon and V.Koch, Phys. Rev. Lett. 85 (2000) 2076.

[19] M.Asakawa, U.Heinz and B.Müller, Phys. Rev. Lett. 85 (2000) 2072.

[20] V.Koch, M.Bleicher and S.Jeon, Phys. Rev. C62 (2000) 061902.

[21] M.Gaździcki, Eur. Phys. J. C8 (1999) 131.

[22] J.Zaranek, preprint hep-ph/0111228 (Nov. 2001)

[23] A.Poskanzer and S.Voloshin, Phys. Rev. C58 (1998) 1671. 


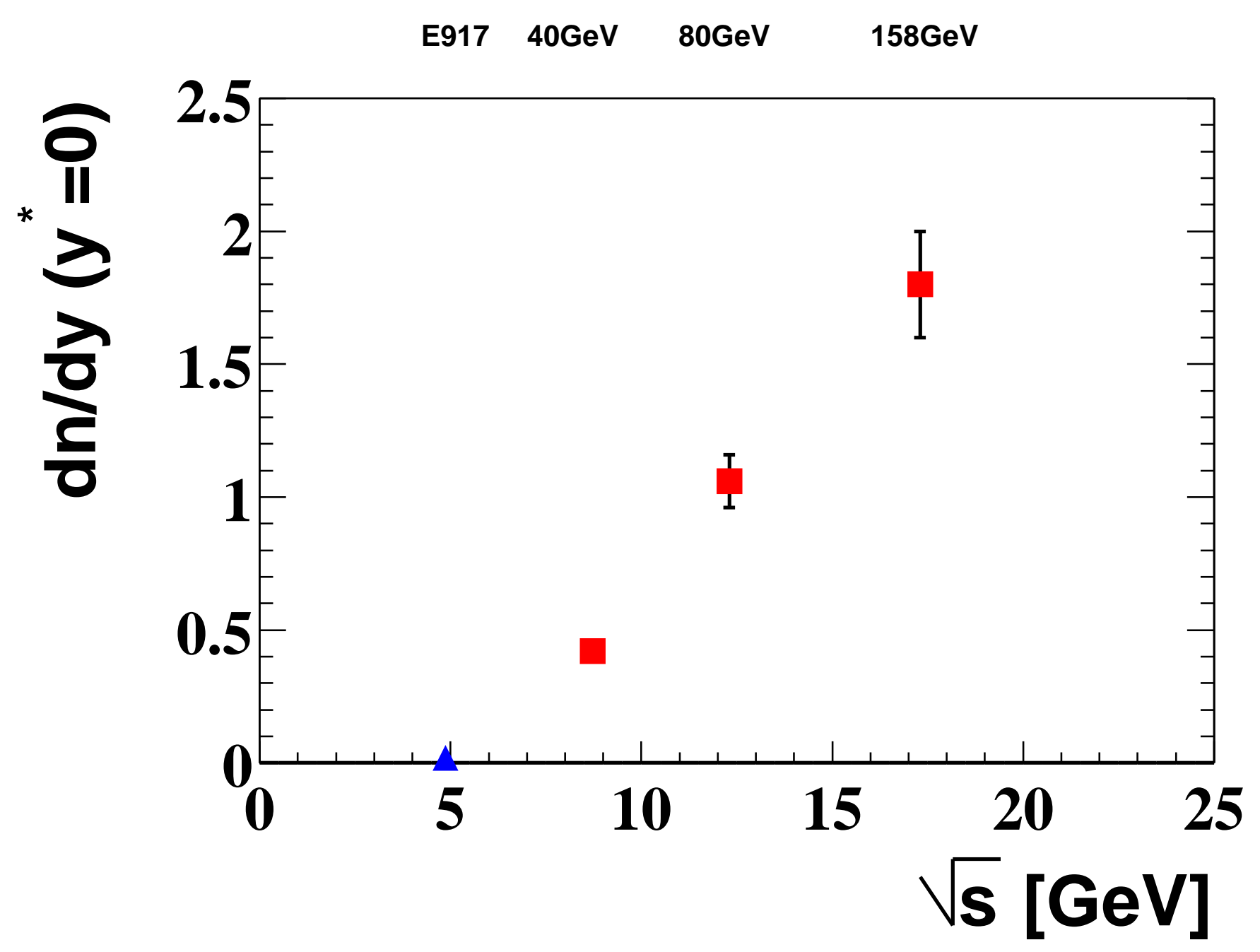




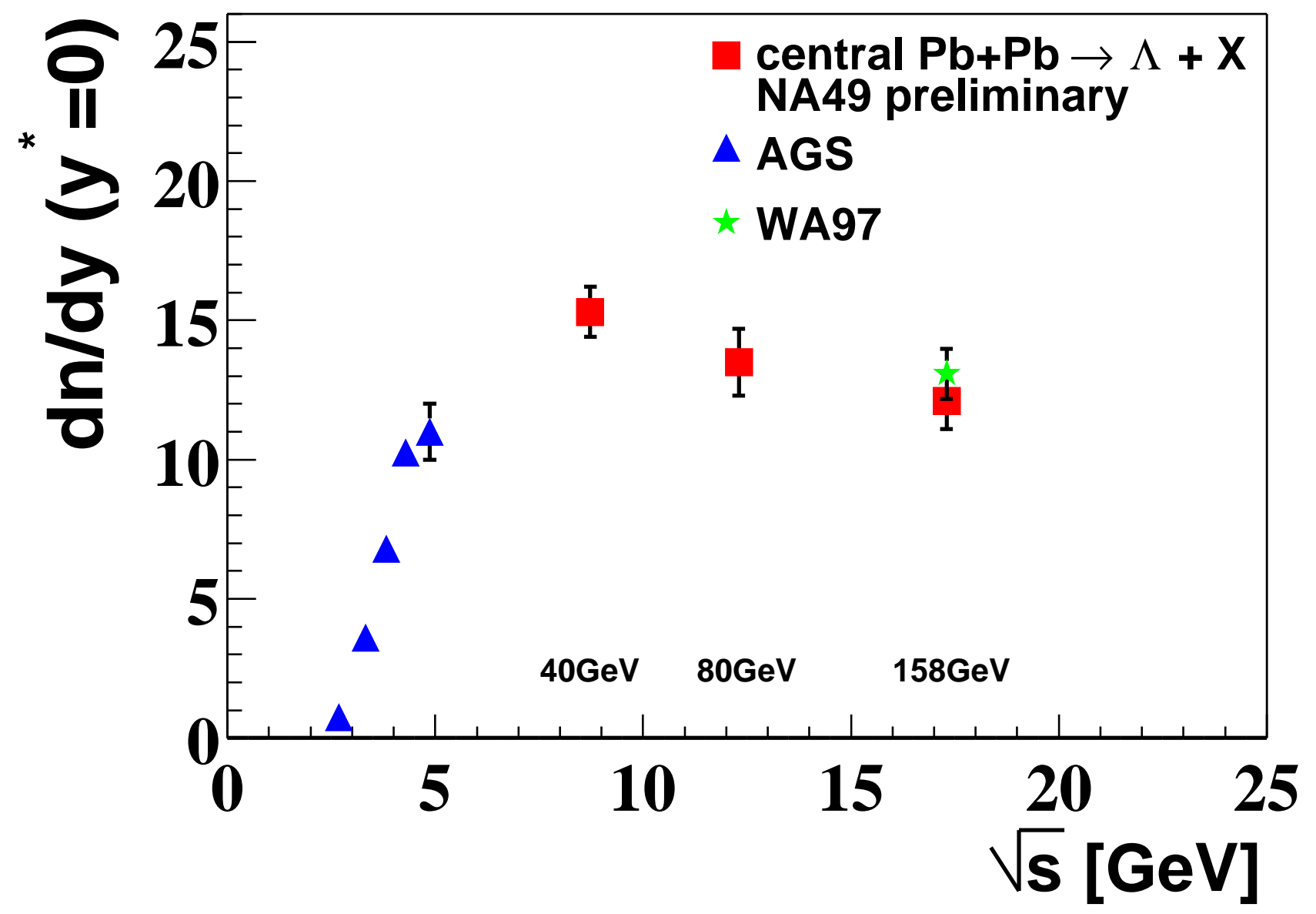

